\title{
Analysis of 2010s Research Trends in Research on Agro-Healing in South Korea
}

\author{
Sun Jin Jeong, Eun Ha Yoo, Jae Soon Kim, Hye Sook Jang, and Geun Woo Lee* \\ Researcher, National Institute of Horticultural and Herbal Science, Wanju-gun 55365, Korea
}

\section{ABSTRACT}

Background and objective: Agro-healing is crucial with urban farming in the domestic. It is beyond the conventional agriculture. This study was carried out to assess the 2010s researches on domestic agro-healing and predict the future direction of agro-healing development.

Methods: Among the articles published from 2010 to 2018, we collected some articles by searching keywords including agro-healing, garden activity, gardening, horticultural activity, horticultural program, horticultural therapy, plant effect, plant environment, plant growing program, plant impact, social gardening, urban agriculture and vegetable garden activity, selected 83 articles that were evaluated in advance, and analyzed by frequency analysis, t-test, and one-way ANOVA with SPSS 20.0.

Results: Agro-healing journal articles were published the most in 2010, and have declined since then. In the classification according to the academic society, most of the journal articles were published by the Society for People, Plants, and Environment. The main targets of domestic agro-healing related to activities and programs were preschoolers, children and adolescents, accounting for $52.4 \%$ of the total. By the characteristics of the subjects, agro-healing programs and studies were conducted with special participants who needed special care compared to the general participants. The dependent variables were classified into six areas according to their attributes and the share of psychological and emotional areas was highest (42.6\%) among them. In terms of the composition of the program, the share of those with 9-12 sessions was highest (36.7\%) and the share of those with more than 20 participants was also highest (39.8\%).

Conclusion: It is recommended to operate agro-healing programs or industries focusing on the socially disadvantaged including those who have special needs or the underprivileged, but in order to create income for farms and expand the demand for agro-healing, it will be necessary to spread the perception that anyone without any physical or emotional issue can be the targets of and experience agro-healing. To meet the different needs of targets of agro-healing, it will be necessary to conduct objective and practical studies on broader areas and in the process the healing functions of agriculture and the strength of agro-healing needs to be further highlighted.

Keywords: garden activities, gardening, plant cultivation, plant environment, urban agriculture

\section{Introduction}

The paradigm of agriculture is being shifted. Along with the government's strategy for promoting the 6th industry, the pluralistic function and value of agriculture and rural areas have been highlighted beyond the conventional production-focused perspective of agriculture and in particular the healing function of agriculture has gained attention (Kim, 2016). This has naturally increased people's interest in agro-healing. Agro-healing is defined as an industry and activities that promote people's psychological, social, cognitive and physical health by utilizing agricultural and rural resources or other associated activities and products (Rural Development Administration, 2016), and all the activities

This study was supported by the research program of the Rural Development Administration (PJ01137605)

Received: October 22, 2019, Revised: November 13, 2019, Accepted: May 5, 2020

First author: Sun Jin Jeong, sunjin75@korea.kr, (i) https://orcid.org/0000-0001-6279-9733

*Corresponding author: Geun Woo Lee, 6606 @naver.com, (D) https://orcid.org/0000-0002-5338-324X 
and products that are directly and indirectly associated with agriculture, natural environments, and cultural or emotional exchanges can be seen as a resource and element of agro-healing (Park and Kang, 2017).

The introduction and activation of agro-healing has a very significant meaning from the perspective of agriculture and society. As changes in industries and social structure have been recently coupled with changes in consumers' demand and the industrial value of agriculture that has long been focusing simply on food production has been weakened, agro-healing can be an alternative for improving the competitiveness of farms, the agents of agricultural activities (Park, 2016). In addition, agro-healing can contribute to reducing social costs by providing caring services suitable for those in need due to physical or emotional illnesses, or the disadvantaged and underprivileged, or the elderly, professional education and training programs connected to the employment and labor market, and services for students or those who need social measures (Gim et al., 2013). Agro-healing activities that utilize plants and nature as a medium were reported to be effective in improving children and adolescents' self-efficacy, psychological wellbeing, self-esteem and sociality and for increasing psychological and emotional relaxation such as reducing aggression, stress and depression (Kang et al., 2012; Keum, 2014; Park and Kim, 2010; Shin, 2010; Soga et al., 2017; Yun and Yoo, 2011). They were also reported to have positive effects on the mental health of adults such as reducing their depression and stress and to be helpful in maintaining their physical health by reducing blood pressure (Jang, Gim, et al., 2019; Jang, Yoo, et al., 2019), and to have other various effects such as improving the mental health of the elderly by reducing a sense of loneliness, depression and stress, improving cognitive functions, the performance of daily life activities and life satisfaction (Kang and Back, 2017; Kim and Yoo, 2010; Lee and Sim, 2004; Oh and Yoo, 2010; Yoo and Jeong, 2010). Seol et al. (2019) proved that activities of producing and selling horticultural products were effective for the vocational rehabilitation of those with mental disorders, and You and Shin (2019) reported that forest experiences can promote the visual perception of children with mental disabilities.

As agro-healing was proved to induce positive physical, psychological and social changes in various groups of people from children to adults, studies on agro-healing have been actively conducted. Therefore, this study aimed to analyze trends in studies on agro-healing published domestically between 2010 to 2018, to suggest the direction for the development of agro-healing in Korea and to provide basic information for follow-up studies by identifying key objects, effective areas and the composition of programs.

\section{Research Methods}

\section{Research objects and data collection}

To achieve the goal of this study - analyzing trends in domestic studies on agro-healing - this study selected as objects earlier research papers that identified the effects of agro-healing utilizing horticultural activities and horticultural healing programs among those published in the journals registered in Korea between 2010 and 2018. Data were collected from the database of the Research Information Service System (RISS) of the Korea Education and Research Information Service (KERIS) using the main keyword agro-healing - with similar words that embrace activities and programs that use agricultural resources as a medium including urban agriculture, garden activity, horticultural activity, vegetable garden activity, effects of plants, environments of plants. These keywords were selected in consultation with researchers in the Agro-healing Research Center of the Urban Agriculture Division of the Rural Development Administration (RDA). The selected studies were found to target various groups of people from children to elderly people, and to use various variables from different areas including physical, psychological, emotional, cognitive and social areas to measure the effects of programs.

\section{Data selection}

A total of 970 research papers were selected by searching keywords, and out of them those that met all the following criteria were selected as the final analysis targets (Table 1). First, considering the qualitative perspective of the research papers, this study targeted research papers published 
Table 1. Number of collected and selected papers by major keywords

\begin{tabular}{cccc}
\hline Searching keyword & Records screened & Records selected & Records excluded \\
\hline Horticultural therapy & 200 & 31 & 169 \\
Horticultural activity & 260 & 25 & 235 \\
Horticultural program & 165 & 13 & 152 \\
Vegetable garden activity & 65 & 5 & 60 \\
Plant growing program & 64 & 3 & 61 \\
Gardening & 2 & 1 & 1 \\
Garden activity & 11 & 1 & 10 \\
Plant environment & 62 & 3 & 59 \\
Plant impact & 4 & 1 & 3 \\
Plant effect & 2 & 0 & 2 \\
Social gardening & 87 & 0 & 87 \\
Urban agriculture & 7 & 0 & 7 \\
Agro-healing & 41 & 070 & 83 \\
Total & & & 81 \\
\hline
\end{tabular}

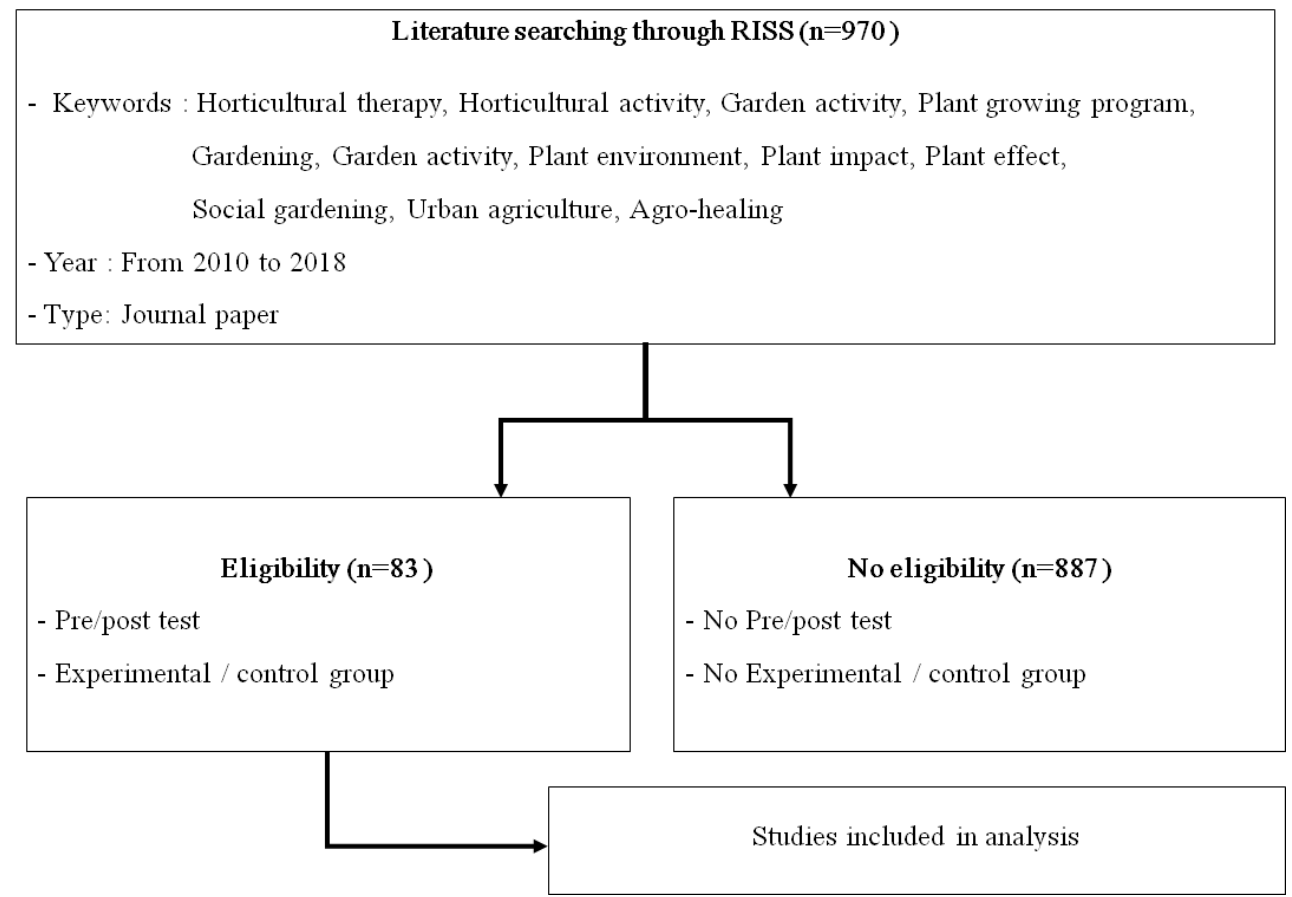

Fig. 1. Literature search and selection process. RISS = Research Information Service System of the Korea Education and Research Information Service.

in the registered journals excluding masters' and doctoral theses. Second, since there are limitations in objectifying and generalizing research results of qualitative studies, only those that utilized statistical quantitative research methods were included in the final analysis objects. Third, only those that conducted both within-subject (pre- and post-tests) and between-subject (experimental and control groups) to verify the effectiveness of programs were included in the final analysis objects. As a result, a total of 83 research papers were selected as the final analysis objects (Fig. 1). 


\section{Data analysis}

The raw data of the final analysis targets were written by entering general information such as the year of publication, the name of authors and the name of journals, information related to research design including the number of participants and dependent variables and information related to programs including the length of the programs and the length of each session. In the process, a total of 117 variables were found to be used to measure the effectiveness of programs including physical activity, depression, learning disorder, interpersonal relationship, hyperactivity, interest in science and life-respect attitude, and they were divided into six categories including physical area, cognitive area, and psychological or emotional area. At first, we intended to adopt the 5-phase life stages (infancy and preschool, childhood and adolescence, young adulthood, middle adulthood, and, late adulthood; Rural Development Administration, 2019) adopted by systems and policies under health and welfare-related laws to divide the life stage of participants. However, since most of the participants in studies that were utilized in analysis were marked as preschoolers, elementary school students, middle and high school students, adults and elderly people, the 5-phase life stages could not be adopted. Instead, they were divided into five life stage groups as follows: preschoolers, children (elementary school students), adolescents (middle and high school students), adults and elderly people. It was because that most of the agro-healing programs conducted on-site were not designed based on the general life stage classification, and in the cases of which participants were mostly students they were in general divided based on education stages (elementary, middle, and high schools). In addition, programs for adults and elderly people were found to be operated without any specific classification of life stage or age. Frequency analysis, $t$-test, and one-way ANOVA were conducted on the collected data utilizing SPSS 20.0.

\section{Results and Discussion}

\section{Trends in research papers on agro-healing}

Trends in research papers on agro-healing that were published between 2010 and 2018 are as follows (Fig. 2). The number of research papers published in 2010 was highest (20.5\%), similar to the results of Park, Lee, Lee, et al. (2016) that the number of research papers on the benefits of horticultural activities for heath drastically increased between 2006 and 2010. The 83 research papers selected to analyze trends in research papers published in journals were found to be published in a total of 22 academic soci-

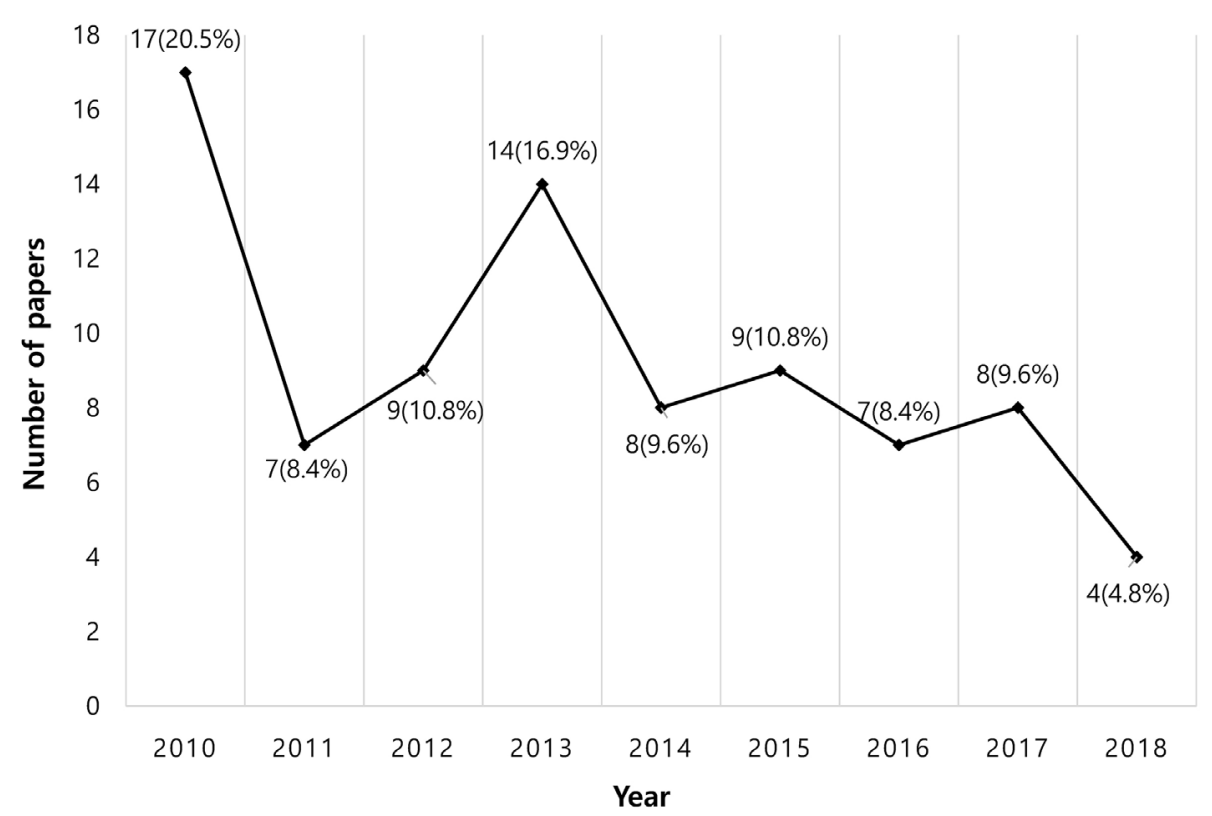

Fig. 2. Changes in the number of published papers in agro-healing by year. 
eties and those that published less than three articles related to agro-healing within the same period were categorized as others. Most research papers on agro-healing were published in the Society for People, Plants and Environment (49.4\%), the Korean Society for Horticultural Science (12.0\%) and the Korean Association of Practical Arts Education (6.0\%), and in particular the Society for People, Plants and Environment accounted for $49.4 \%$ of the analyzed research papers, showing that it published research papers related to agro-healing most actively (Fig. 3).

\section{Classification of characteristics of participants}

To analyze research papers according to the characteristics of participants, they were divided into five life-stage groups and were compared as follows: preschoolers; children (elementary school students); adolescents (middle and high school students); adults; and elderly people. Since some research papers included more than two life-stage groups, multiple response analysis was utilized. As a result (Table 2), the number of research papers that targeted children and adults was $22(25.9 \%)$ respectively, accounting for the highest share, followed by preschoolers $(17.6 \%)$, elderly people (16.5\%) and adolescents (14.1\%). The share of preschoolers, children and adolescents was $57.6 \%$, accounting for the majority, which is similar to the results of Park, Lee, Lee, et al. (2016) that the share of preschoolers, children and adolescents in horticultural activities was $52.4 \%$. These results indicate that research papers published in Korea on agro-healing in the 2010s mainly focused on preschoolers and adolescents. The current aged society is expected to enter a super-aged society in 2026 as the elderly population aged 65 or higher is expected to account for over $20 \%$ of the total population (Korea Institute for Health And Social Affairs, 2019a), and the share is expected to sharply increase to $39.8 \%$ in 2050 (Korea Institute for Health And Social Affairs, 2019b). In light of this, as Park, Lee, Son, et al. (2016) reported that horticultural programs improve the muscle mass, endurance and cognitive ability of elderly people and reduced their

Table 2. Comparison of the number of published papers in agro-healing by subjects' characteristics and life stage

\begin{tabular}{|c|c|c|}
\hline \multicolumn{2}{|c|}{ Variable } & $\mathrm{n}(\%)$ \\
\hline \multirow{2}{*}{$\begin{array}{c}\text { Subject } \\
\text { characteristics }\end{array}$} & General participants & $35(42.2)$ \\
\hline & Special participants & $48(57.8)$ \\
\hline \multirow{5}{*}{$\begin{array}{l}\text { Life stage of } \\
\text { subject }\end{array}$} & Preschoolers & $15(17.6)$ \\
\hline & Children & $22(25.9)$ \\
\hline & Adolescents & $12(14.1)$ \\
\hline & Adults & $22(25.9)$ \\
\hline & Elderly & $14(16.5)$ \\
\hline
\end{tabular}

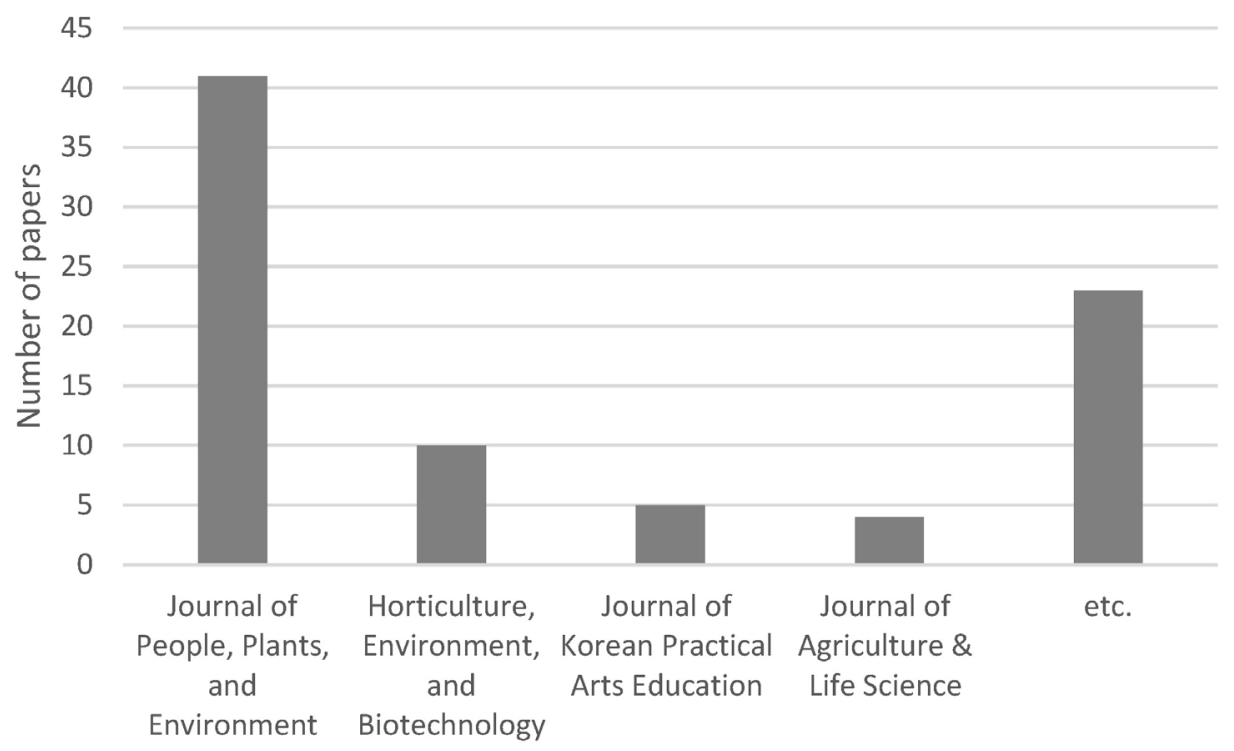

Fig. 3. Comparison of the number of published papers in agro-healing by journal. 
waist line, it will be necessary to develop and expand research on various agro-healing programs for elderly people to maintain and improve their physical and psychological health.

The characteristics of participants were also analyzed by dividing them into general participants and special participants (physically-handicapped persons, patients with stroke and cancer, prisoners, patients with dementia, etc.). The share of general and special participants was $42.2 \%$ and $57.8 \%$ respectively, which shows that the share of special participants was approximately 1.3 times higher. These results indicate that agro-healing programs and research papers on them have been conducted focusing on those who need relatively more help. Therefore, in order to promote the growth of agro-healing programs and related industries, it will be necessary to expand the research and development of various programs targeting general people with the goal of forming healthy life styles, preventing chronic diseases and reducing stress.

Cross-tabulation analysis was conducted on the life stage phases and characteristics of participants and the results were as shown in Table 3. The share of special participants among preschoolers, children and adolescents in the period of childhood and adolescence was higher than that of general participants, while the share of general participants among adults was higher than that of special participants.
In the case of elderly people, the share of general and special participants was equal. Each life stage phase showed different distribution patterns and these differences were statistically significant $\left(\chi^{2}=16.68, p<.01\right)$.

\section{Classification of effect areas according to measured variables}

To analyze tendencies in identifying effects according to measured variables, the six areas suggested by Park, Lee, Lee, et al. (2016) including psychological or emotional, social, cognitive, physical, educational and behavioral areas were referred to for identifying the effects suggested by the research papers analyzed in this study, and finally they were categorized into six areas including physical, psychological or emotional, cognitive, social, educational and others. Since multiple research papers had dependent variables categorized into more than two different subcategories among six categories, their frequency was calculated utilizing multiple response analysis (Table 4). The physical area included hand function, performance of daily life activities and picking up things, and the psychological or emotional area included stress, depression, anxiety and self-esteem. The cognitive area included attention and creativity. The social area included interpersonal relationships, social support and cooperativity, and the educational area included

Table 3. Cross-analysis of the number of published papers in agro-healing by participants' life stage and characteristics

\begin{tabular}{cccccccc}
\hline \multirow{2}{*}{ Characteristic } & \multicolumn{5}{c}{ Life stage of participants } & \multirow{2}{*}{$\chi^{2}(p)$} \\
\cline { 2 - 6 } & Preschoolers & Children & Adolescents & Adults & Elderly & \\
General & 3 & 6 & 4 & 17 & 7 & \multirow{2}{*}{$16.68\left(.002^{* *}\right)$} \\
Special & 12 & 16 & 8 & 5 & 7 & \\
${ }^{* *} p<.01$. & & & & &
\end{tabular}

Table 4. Comparison of the number of published papers in agro-healing by effect area according to dependent variables

\begin{tabular}{ccl}
\hline Effect area & $\mathrm{n}(\%)$ & \\
\hline Physical & $10(7.4)$ & Hand function, activity of daily living, grasping power, gripping ability, etc. \\
Psychological/emotional & $58(42.6)$ & Stress, quality of life, anxiety, depression, happiness, self-efficacy, self-esteem, etc. \\
Cognitive & $10(7.4)$ & Immersion, intellectual development, creativity, attentiveness, etc. \\
Social & $25(18.4)$ & Sociality, relationship, social support, cooperation, reliability, etc. \\
Educational & $8(5.9)$ & Academic achievement, science research ability, achievement motivation, etc. \\
Others & $25(18.4)$ & Salivary cortisol, life-respecting consciousness, job satisfaction, etc. \\
\hline
\end{tabular}


scientific investigation ability and achievement motivation. Other variables were categorized into others. The results of frequency analysis showed that the share of the variables that were categorized into the psychological or emotional area $(42.6 \%)$ and the social area $(18.4 \%)$ were found to be high, while those that set as dependent variables those categorized into the physical area $(7.4 \%)$, the cognitive area $(7.4 \%)$ and the educational area $(5.9 \%)$ were relatively rare. Min et al. (2019) categorized the dependent variables of horticultural activities into five areas including emotional, social, cognitive, physical and linguistic and found that the share of the emotional and social areas was 51\% and $26.3 \%$ respectively and the results of Min et al. (2019) support the results of this study that most variables were categorized into the psychological or emotional and social areas.

To examine differences in effect areas according to the life stage of participants, multiple response cross-tabulation analysis was performed (Table 5), and the frequency of effect areas for each life stage is as follows: preschoolers, psychological or emotional $>$ social $>$ cognitive and educational > physical; children, psychological or emotional $>$ social $>$ educational $>$ cognitive $>$ physical; adolescents, psychological or emotional $>$ social $>$ cognitive $>$ educational and physical; adults, psychological or emotional $>$ social $>$ physical $>$ cognitive and educational; and elderly people, psychological or emotional $>$ physical $>$ cognitive $>$ social $>$ educational. Regardless of the life stage of participants, psychological or emotional variables including stress, depression and self-esteems were found to be used as dependent variables. This indicates that it is necessary to secure diversity in objective and science-based research on a wide range of variables about the healing function of agriculture, and in this process the strength and effectiveness of agro-healing can be highlighted.

\section{Classification according to the composition of programs}

Research papers were divided based on the total number of sessions conducted under programs (Table 6), and the share of those that conducted 9-12 sessions was highest (36.7\%), followed by $13-16$ sessions (30.4\%), 17 sessions or more $(20.3 \%)$, and eight sessions or less $(12.7 \%$; Table $6)$. These results partially coincided with the results of Ji et al. (2010) that surveyed the status of horticultural therapy programs and reported that the share of 11-15 sessions was highest, and can be interpreted in the same vein of the results of Oh (2004) and Kim (2007) that the share of 11-15

Table 5. Comparison of the number of published papers in agro-healing by effect area according to life stage of participants

\begin{tabular}{ccccccc}
\hline \multirow{2}{*}{ Life stage } & \multicolumn{5}{c}{ Effect area } \\
\cline { 2 - 7 } & Physical & Psychological/emotional & Cognitive & Social & Educational & Others \\
\hline Preschoolers & 0 & 5 & 3 & 4 & 3 & 8 \\
Children & 0 & 14 & 3 & 12 & 5 & 8 \\
Adolescents & 0 & 11 & 1 & 3 & 0 & 0 \\
Adults & 6 & 19 & 3 & 1 & 0 & 5 \\
Elderly & 4 & 9 & 3 & 5 & 2 \\
\hline
\end{tabular}

Table 6. Comparison of the number of published papers in agro-healing by the number of sessions in program and life stage of participants

\begin{tabular}{ccccccc}
\hline \multirow{2}{*}{$\begin{array}{c}\text { Number of } \\
\text { sessions }\end{array}$} & Preschoolers & Children & Adolescents & Adults & Elderly & n (\%) \\
\cline { 2 - 6 } & 0 & 0 & 2 & 7 & 1 & $10(12.7)$ \\
8r less & 3 & 9 & 8 & 5 & 4 & $29(36.7)$ \\
$9-12$ & 8 & 6 & 1 & 6 & 3 & $24(30.4)$ \\
$13-16$ & 2 & 3 & 1 & 4 & 6 & $16(20.3)$ \\
17 or over & & & & & &
\end{tabular}


Table 7. Comparison of the number of published papers in agro-healing by the number and life stage of participants

\begin{tabular}{ccccccc}
\hline \multirow{2}{*}{$\begin{array}{c}\text { Number of } \\
\text { participants }\end{array}$} & Preschoolers & Children & Adolescents & Adults & Elderly & $\mathrm{n}(\%)$ \\
\hline & 2 & 5 & 2 & 6 & 4 & $19(22.9)$ \\
9 or less & 2 & 6 & 6 & 10 & 5 & $29(34.9)$ \\
$10-19$ & 11 & 9 & 4 & 5 & 4 & $33(39.8)$ \\
20 or more & & & &
\end{tabular}

sessions was highest. These results also indicate that mid and long-term programs were mainly conducted instead of one-time or short-term programs of 2 months or less, and that programs were mostly terminated within 3-4 months. Cross-tabulation analysis was performed on the age groups of participants and the total number of sessions conducted under programs, and it was found that short-term programs of eight sessions or less mostly were conducted for adults; 9-12 sessions for children and adolescents; 13-16 sessions for preschoolers and children; and 17 sessions or more for elderly people.

Meanwhile, research papers were divided based on the number of participants into less than 10 participants, 10-19 participants and 20 participants or more (Table 7), and the share of 20 participants or more was highest (39.8\%), followed by $10-19$ participants $(34.9 \%)$ and less than 10 participants (22.9). In particular, among the programs for those with developmental disorders, the share of those that targeted 10 participants or less was highest (77.3\%), and among the programs for those with emotional and behavioral disorders, the share of those that targeted 10 participants or less was highest $(84.6 \%)$, which indicates that small-sized healing activities were conducted for those who had special needs (Park, Lee, Lee, et al., 2016).

Cross-tabulation analysis was conducted on the age groups of participants and the number of participants, and it was found that the number of participants in the group of preschoolers and children was relatively higher than other age groups, and these results can be interpreted that most of the research papers that targeted preschoolers and children conducted programs within schools or classes.

\section{Conclusion}

This study aimed to provide basic information on the direction for the development of agro-healing and by analyzing trends in domestic studies on agro-healing. To achieve the objective, this study set criteria for collecting data and selected and utilized in analysis a total of 83 research papers that met the criteria. The results of analysis showed that agro-healing related activities and programs that were conducted in Korea mostly targeted childhood and adolescence that included preschoolers, children and adolescents, and that the number of special participants who had special needs was higher than that of general participants. It was also found that the effects of programs were measured by utilizing mainly the variables in the psychological or emotional area. These results highlights the necessity of developing agro-healing programs for general participants for the spread and industrialization of agro-healing in Korea and proving their effects in various areas other than the psychological or emotional area. It is recommended to operate agro-healing programs and the industry focusing on the socially disadvantaged including those who have special needs or the underprivileged, but in order to create income for farms and expand the demand for agro-healing, it will be necessary to spread the perception that anyone without any physical or emotional issue can be the targets of and experience agro-healing. In addition, over the past decade, studies that were conducted on agro-healing in Korea mostly focused on proving the psychological or emotional effects of agro-healing, and studies have not been sufficiently conducted on other areas. In particular, there were only very few studies on the physical area such as maintaining and improving physical functions. To meet the different needs of targets of agro-healing, it will be necessary to conduct objective and practical studies on broader areas and in the process the healing functions of agriculture and the strength of agro-healing can be further highlighted. At this juncture that people's interest in agro-healing in Korea is growing, one implication of this 
study is that overall trends in studies on agro-healing were analyzed and the direction for the development of agro-healing was suggested in this study.

\section{References}

Gim, G.M., J.Y. Moon, S.J. Jeong, and S.M. Lee. 2013. Analysis on the present status and characteristics of agro-healing in Korea. J. Agric. Ext. Community Dev. 20(4):909-936. https://doi.org/10.12653/jecd.2013.20.4.0909

Jang, H.S., G.M. Gim, S.J. Jeong, and J.S. Kim. 2019. Community gardening activities and their effects on mental health of residents. J. People Plants Environ. 22(4):333-340. https://doi.org/10.11628/ksppe.2019.22.4.333

Jang, H.S., E. Yoo, S.J. Jeong, J.S. Kim, and D.Y. Ryu. 2019. effects of an Agro-healing activity program on the physiological condition of adults with chronic metabolic diseases. J. People Plants Environ. 22(4):355-364. https://doi.org/10.11628/ksppe.2019.22.4.355

Ji, S.E., E.J. Jang, and C.H. Pak. 2010. Analysis of study tendency of Korean masters and doctorate degree dissertations on horticulture therapy. Korean J. Hortic. Sci. Technol. 28(2):328-333.

Kang, H.K. and S.J. Back. 2017. Effect of urban agriculture experience program on the mental health of the elderly. J. People Plants Environ. 20(1):1-6. https://doi.org/10.11 628/ksppe.2017.20.1.001

Kang, S.Y., E.J. Jang, and C.H. Pak. 2012. Effect of horticultural therapy on the self-esteem, social-supporter and stress of the high school students. J. Korean Soc. People Plants Environ. 15(1):9-14.

Keum, J.H. 2014. Effects of arts activities based on plants on psychological well-being and aggression of children. J. Korean Soc. People Plants Environ. 17(1):1-7. https:// doi.org/10.11628/ksppe.2014.17.1.001

Kim, A.R. 2016. In-depth analysis on green care in agriculture of Korea. Master's thesis, Chonnam National University, Gawngju, Korea.

Kim, S.H. 2007. Meta-analysis for the effectiveness of researches related to horticultural therapy. Master's thesis, Konkuk University, Seoul, Korea.

Kim, Y.S. and Y.K. Yoo. 2010. Effects of horticultural therapy on the self-esteem and emotion in older adult with brain lesion. J. Korean Soc. People Plants Environ. 13(2):1-6.

Korea Institute for Health And Social Affairs. 2019a. An analysis of aging policy in international organizations and major pre-experience countries(p. 9). Sejong, Korea: Author.

Korea Institute for Health And Social Affairs. 2019b. Aging population growth and future social policy(p. 9). Sejong, Korea: Author.

Lee, E.S. and W.K. Sim. 2004. Effects of group horticultural activities as a leisure on the isolation and life satisfaction. J. Korean Soc. People Plants Environ. 7(3):31-37.

Min, S.H., M.K. Yun, and J.Y. Kwon. 2019, March. Trends in research on infant horticultural activities. Proceedings of 2019 Spring Annual Conference of the Korean Society for Early Childhood Education (pp. 169-171).

Oh, D.M. 2004. A study on an application of horticultural therapy to social welfare policy. Doctoral dissertation, Jeju National University, Jeju, Korea.

Oh, H.J. and Y.K. Yoo. 2010. Effects of horticultural activity on the social support and self-esteem in solitary old adult. J. Korean Soc. People Plants Environ. 13(4):45-52.

Park, H.C. and D.K. Kang. 2017. Suggestions for applications of therapeutic farms for promoting school community engagement in Korea based on review of therapeutic farms, gardens, animals, policies, and laws in the U.S.A. J. Rural Soc. 27(2):215-269. https://doi.org/10.31894/JR S.2017.12.27.2.215

Park, S.A., A.Y. Lee, G.J. Lee, D.S. Kim, W.S Kim, C.A. Shoemaker, and K.C. Son. 2016. Horticultural activity interventions and outcomes: A review. Korean J. Hortic. Sci. Technol. 34(4):513-527. https://doi.org/10.12972/kj hst. 20160053

Park, S.A., A.Y. Lee, K.C. Son, W.L. Lee, and D.S. Kim. 2016. Gardening intervention for physical and psychological health benefits in elderly women at community centers. HortTechnology 26(4):474-483. https://doi.org/ 10.21273/HORTTECH.26.4.474

Park, S.Y. 2016. Basic scheme for systematic introduction of green care in agriculture in Korea. Doctoral dissertation, Chonnam National University, Gawngju, Korea.

Park, Y.H. and H.Y. Kim. 2010. Effect of horticultural 
therapy on the self-efficacy and emotional intelligence of children in the child welfare institutions. J. Korean Soc. People Plants Environ. 13(2):7-11.

Rural Development Administration. 2016. Understanding of agro-healing or care farming(pp. 10-13). Jeonju, Korea: Author.

Rural Development Administration. 2019. Design of agro-healing service in agriculture considering life cycle(pp. 6-10). Jeonju, Korea: Author.

Seol, G.A., S.Y. Yun, and B.J. Choi. 2019. The effects of making horticultural products and selling activities on vocational rehabilitation of persons with mental disabilities. J. People Plants Environ. 22(3):279-287. https://doi.org/10.11628/ksppe.2019.22.3.279

Shin, Y.S. 2010. Effect of after school horticultural activity on the sociality and self-esteem of children from the low income families. J. Korean Soc. People Plants
Environ. 13(6):33-39.

Soga, M., K.J. Gaston, and Y. Yamaura. 2017. Gardening is beneficial for health: A meta-analysis. Prev. Med. Rep. 5:92-95. https://doi.org/10.1016/j.pmedr.2016.11.007

Yoo, Y.K. and H.Y. Jeong. 2010. Effect of horticultural therapy program on the depression and self-esteem of the older adult in facilities. J. Korean Soc. People Plants Environ. 13(3):7-15.

You, J.H. and W.S. Shin. 2019. The effects of psychomotorik in the forest on the sensory and visual perception of children with intellectual disabilities. J. People Plants Environ. 22(3):321-332. https://doi.org/10.11628/ksppe. 2019.22.3.321

Yun, E.J. and Y.K. Yoo. 2011. Effects of horticultural activity on the mental health, sociality in children from low income family. J. Korean Soc. People Plants Environ. 14(1):17-22. 